\title{
Novel approaches for the molecular classification of prostate
} cancer

\author{
Robert H. Getzenberg \\ Department of Urology, James Buchanan Brady Urological Institute, The Johns Hopkins University School of Medicine, \\ Baltimore 21287, MD, USA
}

Asian Journal of Andrology (2010) 12: 620-622. doi: 10.1038/aja.2010.95; published online 16 August 2010.

Among the urologic cancers, prostate cancer is by far the most common, and it appears to have the potential to affect almost all men throughout the world as they age. A number of studies have shown that many men with prostate cancer will not die from their disease, but rather with the disease but from other causes. These men have a form of prostate cancer that is described as "very low risk" and has often been called indolent. There are however a group of men that have a form of prostate cancer that is much more aggressive and life threatening. Unlike other cancer types, we have few tools to provide for the molecular classification of prostate cancer. This limits the potential to personalize treatment approaches for the disease. Several recent studies have focused on attempting to provide a better classi-

Correspondence to: Prof. Robert H. Getzenberg, Department of Urology, James Buchanan Brady Urological Institute, The Johns Hopkins University School of Medicine, Baltimore 21287, MD, USA.

Fax: +1-410-502-9336

E-mail: rgetzen1@jhmi.edu fication of prostate cancer in order to differentiate the types of the disease as well as to provide novel treatment targets. These approaches have utilized the latest technologies and sequencing capabilities to accomplish this goal. Two recent studies by Taylor et al. [1] and Yu et al. [2] are good examples of these approaches. Understanding the significance of these studies and their potential applications to prostate cancer is critical to reaching a better definition of the disease which impacts so many men.

Taylor et al. [1] examined global DNA copy numbers (i.e. the numbers of copies of each gene) along with expression profiles (mRNAs that are expressed) to examine 218 prostate cancer tumors, both from individuals with localized disease as well as those with metastases. In other tumor types including lung cancer, breast cancer and glioblastoma, genomic alterations have been relatively easily identified. However, from this study by Taylor et al. [1] it appears that point mutations (single changes in the DNA sequence) may occur only rarely in prostate cancers. In fact, as the authors themselves conclude in their paper, there are no single genes that are commonly mutated in prostate cancer. Genes that are known to be altered in prostate cancer-for example, $p 53$ and the tumor suppressor PTEN-are altered not by mutation, but by copy number changes. However, this comprehensive study did reveal several interesting modifications of the androgen pathway that seem to be important in prostate cancer. A co-activator of the androgen receptor (AR) known as NCOA2 was shown to be altered in a number of the prostate cancers studied. Functional studies demonstrated that altering $N C O A 2$ levels may provide additional outputs to the androgen pathway and, therefore, has the potential to drive the disease. Interestingly, rather than finding actual changes to the AR, they observed changes in co-factors like NCOA2 and $N C O R 2$ in localized primary tumors.

Another interesting change observed by the authors in their study was that an area on chromosome 3 (3p14) appears to be deleted in a number of prostate cancers. Three 
interesting genes $F O X P 1, R Y B P$, and $S H Q 1$ lie within this region. These have the potential to serve as tumor suppressors or other kinds of regulators that the absence of which appears to be important in the development of prostate cancer. Overall, it is interesting to note the lack of consistent and robust genetic changes that are found within prostate cancer. This seems to represent the diverse nature of the disease and may further demonstrate the importance of developing strategies to subclassify prostate cancers into distinct entities.

Overall, the strengths of the study by Taylor et al. [1], include the comprehensive nature of the genomic analysis that is performed. This is supported by strong clinical data that provides meaning to the studies being performed. Finally, the population size examined is reasonable. Some of the limitations to the study outlined include the fact that the authors included, did not look at truly normal individuals. They therefore do not know which genetic alterations may indeed be associated with prostate cancer versus those truly without the disease. They also focused on relatively large tumor sizes. In doing so, they may have missed some important smaller lesions that could contain signatures with the potential to drive prostate cancer to a more aggressive state. With the known multi-focality of the disease, examining the various tumors within the prostate may be important in gaining an understanding of the true genetic basis of the disease. For example, another study by Miller and colleagues [3] demonstrated that many of the smaller lesions within the prostate have the same genetic signature as the resulting metastases unlike some of the larger lesions that are typically studied. Despite these limitations, however, the study is important as it reveals that prostate cancer is a complex disease that will not be as easy to decipher as a number of other cancers have been, at least at the genomic level.

In an earlier issue of Cancer Cell, investigators from the University of Michigan utilized used a different approach to try to better decipher the genetic pathways associated with the progression of prostate cancer. In this case, Yu et al. [2] have utilized a technique known as chromatin immunoprecipitation (ChIP) together with extensive sequencing to decipher new aspects of not only the identified gene fusion of TMPRSS2 to several members of the ETS transcription factor family. These gene fusions have been observed in a large percentage of prostate cancers. The researchers studied prostate cancer cell lines and a single prostate cancer specimen.

Chromatin immunoprecipitation utilizes antibodies to pull out specific proteins and their associated DNA sequences. The sequences are then analyzed to determine the specific genes that might be associated with individual proteins. For example, here the researchers looked at the AR to determine the associated sequences. They also looked at several other genes that are known to be important in prostate cancer: ERG, FoxAl and RNA $P O L I I$, along with the sequences that are associated with the genes and which may be specific for prostate cancer. They identified a number of genes that have previously been shown to be associated with the AR. In addition to the canonical ARE sequence, the next most abundant set of sequences associated with the AR were those belonging to the ETS transcription factor family. This finding coincides nicely with previously identified gene fusions to demonstrate the androgen regulation of this family of factors in prostate cancer. Finally, the authors showed that ERG is associated with more than $90 \%$ of the $A R$ genes. These findings illustrate the relationship between the pathways of ERG and the AR.

The investigators proposed that the TMPRSS2-ERG gene fusion alters the androgen pathway and connects the androgen pathway to the Polycomb group of proteins. They went on to suggest that therapies targeting the AR may not be effective, that the true driver of prostate cancer may be the fusion of TMPRSS2-ERG, and that higher doses of testosterone may be more therapeutically effective than hormonal withdrawal. This study extensively catalogues the transcription factors and system modifications that are associated with prostate cancer, a data-set that will be extremely valuable for future studies.

The major strength of the work by $\mathrm{Yu}$ et al. [2] is the comprehensive nature by which they studied the association between the AR, the Polycomb network and the TMPRSS2-ERG gene fusion. They used a number of prostate cancer cell lines to conduct their investigations and their results highlight the importance of TMPRSS2-ERG fusion in prostate cancer, suggesting a direct role in the regulation of castrate-resistant prostate cancer. One of the limitations of this work is that it focuses almost entirely on 
prostate cancer cell lines as models and does not involve interrogation of human samples beyond a single prostate cancer specimen. Another limitation is that TMPRSS2-ERG gene fusions have generally been found to be associated with earlier stage prostate cancer rather than the later stage disease that would be more typically associated with castrate-resistant prostate cancer. Therefore, the clinical significance of these findings still needs to be demonstrated.

These two papers represent comprehensive and unique analyses of the genomic alterations and pathways that are associated with prostate cancer. Using state-of-theart approaches these investigators have delved deeply into prostate cancer to begin to dissect out some of the critical pathways and components that are associated with the disease. Their conclusions support the idea that prostate cancer is a complex disease for which simple answers will not be forthcoming. At the same time, we are beginning to get clues about the critical regulators that may be involved in the development of the disease as well as its progression to a more malignant phenotype. Future studies will certainly build upon these fundamental studies as we continue to understand more about a disease that affects almost all men as they age.

\section{References}

1. Taylor BS, Schultz N, Hieronymus $\mathrm{H}$, Gopalan A, Xiao Y, et al. Integrative genomic profiling of human prostate cancer. Cancer Cell 2010; 18: 11-22. Epub 2010 Jun 24.

2. Yu J, Yu J, Mani RS, Cao Q, Brenner $\mathrm{CJ}$, et al. An integrated network of androgen receptor, polycomb, and TMPRSS2-ERG gene fusions in prostate cancer progression. Cancer Cell 2010; 17: 443-54.

3. Ruijter ET, Miller GJ, van de Kaa CA, van Bokhoven A, Bussemakers $\mathrm{MJ}$, et al. Molecular analysis of multifocal prostate cancer lesions. J Pathol 1999; 188: 271-7. 